\title{
Color preference in squirrel monkeys (Saimiri sciureus)
}

KENNETH F. GREEN, JOHN W. MOORE AND THEODORE D. SARGENT

UNIVERSITY OF MASSACHUSETTS, AMHERST

Eight naive squirrel monkeys were found, by the paired comparisons method, to prefer darker-hued colors.

The purpose of this experiment was to find, prior to color discrimination training, if monkeys were biased towards any particular color or part of the color spectrum and, if so, to determine the strength of the bias.

Method

The apparatus used was a sheet metal cubicle which contained two display panels mounted side by side in one wall (Fig. 1). Each display panel contained a pair of holes behind which the stimuli could be mounted and a third hole for access to a delivery cup. A pair of guillotine screens covered each display panel: the outer screen was opaque to prevent $S$ from seeing the discriminanda between trials and the inner one was transparent to prevent $S$ from touching the stimuli prior to a brief exposure period at the start of a trial. The display panels were flat white, the opaque screens flat gray, and the remainder of the apparatus was flat black. Stimuli were colored cards which served also as manipulanda.

Eight naive adult squirrel monkeys $(4$ males and 4 females) were trained to press the stimulus cards by an approximation method. After magazine training, the Ss were required in successive stages to press a transparent card behind which a pellet was shown, to press an opaque card which had a pellet lodged between its base and its holder, and finally to press an opaque card with no pellet present. Each pretraining press resulted in delivery of a CIBA $75-\mathrm{mg}$ banana pellet into the nearest food cup.

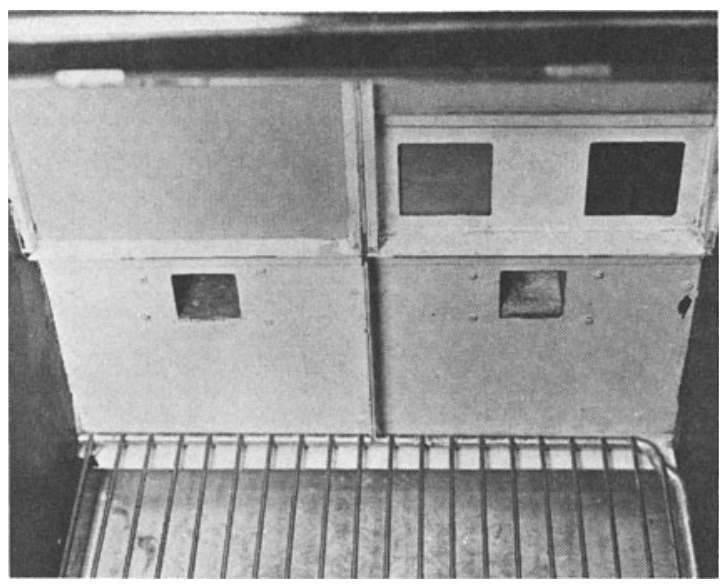

Fig. 1. The interior of the discrimination apparatus with guillotine screens raised to show one display panel and a pair of stimuli.

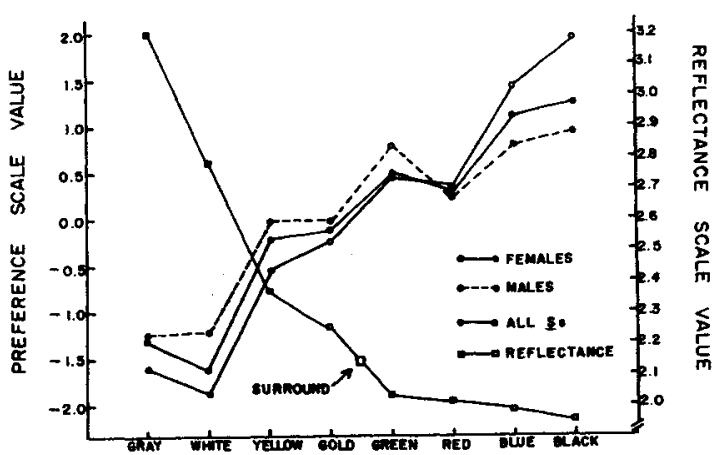

Fig. 2. Results of the first preference test, showing color preference scales for males, females, and both groups combined, and reflectance curve for the eight colored stimuli. Preference values are standard deviations and reflectance values are averaged readings from an exposure meter scale.

The color preference test was administered by the paired comparisons method. The stimulus colors were Pleasuretone enamel made by Star Bronze Co., Alliance, Ohio, and in order of reflectance as measured under standard conditions were gray, white, yellow, gold, green, red, blue, and black (cf. Fig. 2). Although nearly indistinguishable in terms of reflectance, the latter four stimuli were easily distinguishable to the monkeys, hence, although technically incorrect, all colors as listed above will be referred to as lying on a dimension from bright-hued to dark-hued. The reflectance of the display panels was intermediate between gold and green. Each possible pair of different colors was presented four times in a random order which allowed counterbalancing of the side of the apparatus on which the pair was presented and the position of each stimulus within the pair. All 112 responses in the preference test were rewarded.

A second test for color preference was given after completion of a conditional-outcome choice experiment (Green \& Moore, 1966). Six monkeys which had not position responded for more than 10 consecutive days in that experiment were used as Ss. The procedure was identical to that in the first experiment, but the display panels and guillotine screens were painted flat black and the remaining walls of the apparatus were painted flat white in an attempt to find if the original preferences were based on color or on contrast between the stimuli and the surround.

Results

Pretraining required 30-60 days, and the females took nearly twice as long as the males to learn the panel press response. 
In the first preference test, seven $\mathrm{Ss}$ position responded on $61-74 \%$ of the trials, and one male position responded on $90 \%$ of the trials. In Fig. 2 are shown preference scales for the males, females, and both groups combined, constructed according to procedures given by Torgerson (1958, pp. $168 \mathrm{ff}$.) Thurstone's Case IV was used instead of the more popular Case $\mathrm{V}$ because the discriminal dispersions of the stimuli were unequal. The scales show that the darker-hued the color the greater the preference. Also, the preferences of the females tended to be stronger than those of the males. One notable inversion of preference occurred at the red stimulus, with the females appearing more "averse" to this color than the males.

In the second preference test, position responding increased in all animals such that the lowest percentage was $76 \%$ responses to the preferred side. The tendency to prefer darker-hued colors was still present, but, in that only $52.3 \%$ of the responses were made to the four darkest-hued stimuli, the preference was greatly diminished and far less clear-cut than in the first test. Discussion

It may be questioned whether the obtained preferences were based on color or on reflectance. Insofar as the darkest-hued stimuli were nearly indistinguishable in terms of the reflectance measure and the animals differentiated among them quite nicely in terms of the preference measure, the preferences appear to be based on color. In addition, contrast between the stimuli and the surround did not appear to affect preference because the preference curve in the first test was symmetrical about the middle reflectance value (that of the surround) and because the preferences in the second test were grossly similar to those in the first test. Corroboration of the present result is offered by Yerkes' finding that apes prefer blues and greens to yellows, oranges, and reds (Harlow, 1945), and the fact that innate color preferences have been reported in other vertebrates (Hess, 1956). Thus, our finding that squirrel monkeys prefer some colors to others should be taken into account in situations where colored stimuli are used.

\section{References}

Green, K. F., \& Moore, J. W. Conditional-outcome choice behavior in squirrel monkeys. Psychon, Sci., 1966, 4,

Harlow, H. F. Studies on discrimination learning by monkeys: VI. Discrimination between stimuli differing in both color and form, only in color, and only in form. J. gen. Psychol., 1945, 33, 225235 .

Hess, E. H. Natural preferences of chicks and ducklings for objects of different colors. Psychol. Rep., 1956, 2, 477-483. 